\title{
Tyrosine Kinase Pyk2 is Involved in Colonic Smooth Muscle Contraction via the RhoA/ROCK Pathway
}

\author{
Ling TONG ${ }^{1,3}$, Jun-Ping AO ${ }^{2}$, Hong-Li LU ${ }^{1}$, Xu HUANG ${ }^{1}$, Jing-Yu ZANG ${ }^{3}$, Shao-Hua LIU $^{4}$, \\ Ni-Na SONG ${ }^{1}$, Shi-Qi HUANG ${ }^{1}$, Chen Lu ${ }^{1}$, Jie CHEN ${ }^{2}$, Wen-Xie XU ${ }^{1,3}$ \\ ${ }^{1}$ Department of Anatomy and Physiology, Shanghai Jiao Tong University School of Medicine, \\ Shanghai, China, ${ }^{2}$ State Key Laboratory of Oncogenes and Related Genes, Shanghai Cancer \\ Institute, Renji Hospital, Shanghai Jiao Tong University, School of Medicine, Shanghai, China, \\ ${ }^{3}$ Department of Pediatric Surgery, Xin Hua Hospital, Affiliated with Shanghai Jiao Tong University \\ School of Medicine, Shanghai, China, ${ }^{4}$ Department of Anesthesiology, Soth Renji Hospital, \\ Affiliated with Shanghai Jiao Tong University School of Medicine, Shanghai, China
}

Received January 7, 2018

Accepted July 26, 2018

Epub Ahead of Print October 23, 2018

\section{Summary}

The contraction of gastrointestinal (GI) smooth muscles is regulated by both $\mathrm{Ca}^{2+}$-dependent and $\mathrm{Ca}^{2+}$ sensitization mechanisms. Proline-rich tyrosine kinase 2 (Pyk2) is involved in the depolarization-induced contraction of vascular smooth muscle via a $\mathrm{Ca}^{2+}$ sensitization pathway. However, the role of Pyk2 in GI smooth muscle contraction is unclear. The spontaneous contraction of colonic smooth muscle was measured by using isometric force transducers. Protein and phosphorylation levels were determined by using western blotting. Pyk2 protein was expressed in colonic tissue, and spontaneous colonic contractions were inhibited by PF-431396, a Pyk2 inhibitor, in the presence of tetrodotoxin (TTX). In cultured colonic smooth muscle cells (CSMCs), PF-431396 decreased the levels of myosin light chain (MLC 20 ) phosphorylated at Ser19 and ROCK2 protein expression, but myosin light chain kinase (MLCK) expression was not altered. However, Y-27632, a Rho kinase inhibitor, increased phosphorylation of Pyk2 at Tyr402 and concomitantly decreased ROCK2 levels; the expression of MLCK in CSMCs did not change. The expression of P(Tyr402)-Pyk2 and ROCK2 was increased when CSMCs were treated with Ach. Pyk2 is involved in the process of colonic smooth muscle contraction through the RhoA/ROCK pathway. These pathways may provide very important targets for investigating GI motility disorders.

\section{Key words}

Pyk2 (proline-rich tyrosine kinase 2) - RhoA/ROCK pathway • $\mathrm{Ca}^{2+}$-sensitization $\bullet$ Colon smooth muscle $\bullet$ Contraction

\section{Corresponding authors}

W.-X. Xu, Department of Anatomy and Physiology, College of Basic Medicine, School of Medicine, Shanghai Jiao Tong University, 800 Dong Chuan Road, Room 328, Wenxuan Medical Building, 200240 Shanghai, China. E-mail: wenxiexu@sjtu.edu.cn and J. Chen, Department of Pediatric Surgery, Xin Hua Hospital, Affiliated with Shanghai Jiao Tong University School of Medicine, 1665 Kong Jiang Road, 200092 Shanghai, China. E-mail: jiechen1974@163.com

\section{Introduction}

Gastric motility requires interactions between smooth muscle, enteric and extrinsic autonomic nerves, and the intestinal cells of Cajal (ICC) (Grover et al. 2011). Smooth muscle cells (SMCs) are considered the final effector cells responsible for producing fine and delicate gastrointestinal (GI) tract movement (Alshboul 2015). The contractile response of GI smooth muscle is primarily mediated by the phosphorylation of myosin regulatory light chain $\left(\mathrm{MLC}_{20}\right)$ at Ser19 (Somlyo and Somlyo 2003), and the degree and duration of contraction depend on the balance between the activity of myosin light chain kinase (MLCK) and myosin light chain phosphatase (MLCP) (Hartshorne et al. 2004, Sun et al. 2015). Smooth muscle cells generate depolarization when they are stimulated. The intracellular $\mathrm{Ca}^{2+}$ concentration $\left(\left[\mathrm{Ca}^{2+}\right]_{\mathrm{i}}\right)$ is elevated by a $\mathrm{Ca}^{2+}$ influx from the 
extracellular space via ion channels, where the most important channels are voltage-operated $\mathrm{Ca}^{2+}$ channels (VOCCs). The elevation of $\left[\mathrm{Ca}^{2+}\right]_{\mathrm{i}}$ increases the levels of $\mathrm{Ca}^{2+}$-bound calmodulin, which then activates MLCK followed by phosphorylating $\mathrm{MLC}_{20}$ at Ser19, resulting in forceful muscle contraction (Somlyo and Somlyo 2003). This pathway is a classic $\mathrm{Ca}^{2+}$-dependent pathway, which plays a predominant role in initiating contraction (Somara et al. 2007). However, in addition, membrane depolarization also induces the activation of G-proteincouple receptors, which results in activation of the RhoA/Rho kinase pathway (RhoA/ROCK pathway) and the protein kinase $\mathrm{C}$ pathway, which inhibit the activity of MLCP and favors the phosphorylation of $\mathrm{MLC}_{20}$ (Kitazawa et al. 1991, Somara et al. 2007, Alshboul 2015). In these processes, the smooth muscle forces are enhanced without a change in $\left[\mathrm{Ca}^{2+}\right]_{i}$, and this phenomenon is called the $\mathrm{Ca}^{2+}$-sensitization pathway (Kitazawa et al. 1991). A sustained contractile response has been suggested to be associated with this process.

Pyk2 (proline-rich tyrosine kinase 2) is a nonreceptor tyrosine kinase (Bayer et al. 2003, Lipinski and Loftus 2010), which is highly expressed in cells of the hematopoietic lineage and in the central nervous system (Avraham et al. 2000). Regarding the intracellular distribution, Pyk2 is often enriched in the perinuclear region (Zhang et al. 2008). Various stimuli, including neurotransmitters, bioactive lipids, growth factors, and increased intracellular calcium concentrations, induce Pyk2 phosphorylation. In particular, tyrosine 402 (Tyr-402) is the first residue to be autophosphorylated and represents the initial event in Pyk2 activation; activated Pyk2 then influences multiple signaling pathways, such as the MAP kinase signaling pathway and the Rho kinase pathway (Wu et al. 2006, Lipinski and Loftus 2010). It has been reported that Pyk2 is phosphorylated at Tyr402 and involved in the contractile response via the RhoA/ROCK signaling pathway in depolarization-induced contraction of vascular smooth muscle cells (VSMCs) (Mills et al. 2015). In addition, angiotensin II (Ang II) induces contraction responses of VSMCs by activating the RhoA/ROCK signaling pathway (Rattan et al. 2003, Wang et al. 2015), and some researchers think that Pyk2 is activated and associated with the RhoA/ROCK signaling pathway in AngIIinduced contraction of VSMCs and that the activation of RhoA is reduced when the activity of Pyk2 is inhibited (Ying et al. 2009b). Vascular smooth muscle and GI smooth muscle are both smooth muscle; however, whether Pyk2 plays a similar role in the contraction of GI smooth muscle has not been reported previously.

Intestinal motility dysfunction conditions are very common, especially in colon. Therefore, in the present study, we hypothesized that the role of Pyk2 during the contraction of colon smooth muscle is the same as in vascular smooth muscle, which is activated by intracellular calcium and related to the contractile response through the RhoA/ROCK signaling pathway. Here, Pyk2 was shown to be associated with the RhoA/ROCK signaling pathway in the contraction response of colonic smooth muscle, a mechanism that is similar to that seen in vascular smooth muscle.

\section{Methods}

\section{Ethics}

This study was conducted in strict accordance with the recommendations in the Guide for the Care and Use of Laboratory Animals of the Science and Technology Commission of P.R.C. (STCC Publication No. 2, revised 1988). The protocol was approved by the Committee on the Ethics of Animal Experiments of Shanghai Jiaotong University School of Medicine (Permit Number: Hu 686-2009).

\section{Animals}

Adult male ICR mice aged 4 weeks weighing 20-25 g were purchased from the Experimental Animal Center of the Chinese Academy of Sciences, Shanghai, China. The mice were housed at a constant temperature $\left(20-25^{\circ} \mathrm{C}\right)$ under a $12 \mathrm{~h} \mathrm{light/dark} \mathrm{cycle,} \mathrm{with} \mathrm{free} \mathrm{access}$ to water and food.

\section{Solutions and drugs}

Tissues were maintained in Krebs solution $(\mathrm{mmol} / \mathrm{l}): \quad \mathrm{NaCl}$ 121.9, $\mathrm{NaHCO}_{3}$ 15.5, $\mathrm{KCl}$ 5.9, $\mathrm{MgSO}_{4} 1.2, \mathrm{KH}_{2} \mathrm{PO}_{4} 1.2$, glucose 11.5 , and $\mathrm{CaCl}_{2} 2.5$. Tetrodotoxin citrate (TTX), Bay K8644 and acetylcholine chloride (Ach) were purchased from Tocris Bioscience (Bristol, UK). Y-27632, PF-431396, and nifedipine were purchased from Sigma Aldrich (St. Louis, MO, USA).

\section{Muscle strip preparation and isometric tension measurements}

Mice were euthanized by cervical dislocation, and a segment of the colon was immediately removed and placed in aerated $\left(95 \% \mathrm{O}_{2}\right.$ and $\left.5 \% \mathrm{CO}_{2}\right)$ Krebs solution. Then, after the mesentery was removed with tweezers, 
the colon was cut open along the mesenteric border and pinned to the base of the Sylgard dish with the mucosa facing upward, and the mucosa and submucosa were removed. The isolated colon muscle strips $(2 \mathrm{~mm} \times 8 \mathrm{~mm})$ were longitudinally mounted in an organ bath. A silk thread (USP 5/0) was attached to both ends of the strips. One end of each strip was fixed to the bottom of the muscle chamber, and the other end was attached to an isometric muscle transducer for continuous monitoring of isometric tension. The organ bath was filled with $8 \mathrm{ml}$ of warm $\left(37^{\circ} \mathrm{C}\right) \mathrm{Krebs}$ solution and bubbled continuously with $95 \% \mathrm{O}_{2}$ and $5 \% \mathrm{CO}_{2}$. Each mouse muscle strip was placed under a preload of $0 \mathrm{~g}$. The tension energy transducer transmitted the tension signal into the physiological recorder of an RM6280 multi-channel physiological signal acquisition processing system (Instrument Company of Chengdu, Chengdu, China). After the muscle strips were equilibrated in the chamber for $30 \mathrm{~min}$, the dimensional displacement spinner was adjusted to increase the muscular tension to $0.3 \mathrm{~g}$. The experimental drugs were administered when the amplitude and frequency of spontaneous muscle strip contractions were stable.

\section{Colonic smooth muscle cell culture}

The colon was opened along the mesenteric border, and the mucosa and submucosa were removed. The layer of colonic smooth muscle was washed with germ-free PBS, cut into several small pieces, placed in a six-well plate containing Dulbecco's Modified Eagle's Medium (DMEM) supplemented with $10 \%$ heatinactivated fetal bovine serum (FBS), $100 \mathrm{U} / \mathrm{ml}$ penicillin and $100 \mathrm{mg} / \mathrm{ml}$ streptomycin, and cultured in a $37^{\circ} \mathrm{C}$ incubator with a $5 \% \mathrm{CO}_{2}$ atmosphere. After one week in culture, the cells displayed a triangular or flat-shaped morphology. CSMCs were used between the fourth and six passages for the study. Cells were incubated in serumfree DMEM for $24 \mathrm{~h}$ prior to the drug treatments.

\section{Cell lysis and western blotting}

Protein samples were extracted from the CSMCs. Cells that had been incubated in the absence or presence of various drugs were washed three times with ice-cold phosphate-buffered saline (PBS), lysed with RIPA buffer (P0013, Beyotime Chemical Co., Jiangsu, China) containing protease inhibitors (P8340, Sigma, MO, USA) and phosphatase inhibitors (P0044, Sigma, MO, USA), and then incubated on ice for $20 \mathrm{~min}$. Cell lysates were centrifuged at $12,000 \mathrm{x}$ g for $20 \mathrm{~min}$ at $4{ }^{\circ} \mathrm{C}$.
Equivalent amounts of protein (normally $40 \mu \mathrm{g}$ per lane) were then subjected to $8 \%$ or $10 \%$ sodium dodecyl sulphate polyacrylamide gel electrophoresis (SDS-PAGE) and transferred to polyvinylidene difluoride (PVDF) membranes (Millipore, MA, USA). PVDF membranes were blocked in Tris-buffered saline-Tween 20 (TBS-T) containing $5 \%(\mathrm{w} / \mathrm{v})$ non-fat dry milk for $120 \mathrm{~min}$ at room temperature. Membranes were incubated with an anti-Pyk2 antibody (1:1,000; SAB4500837, Sigma), anti-p-Tyr402-Pyk2 antibody (1:1,000; 44168G, Invitrogen), anti-phospho-myosin light chain (Ser19) antibody (1:1,000; \#3671, Cell Signaling Technology), anti-myosin light chain 2 antibody (1:1,000; \#3672, Cell Signaling Technology), anti-phosphor-MYPT1 (Thr853) antibody (1:1,000; \#4563, Cell Signaling Technology), anti-MYPT1 antibody $(1: 1,000$; \#2634, Cell Signaling Technology), anti-ROCK1 antibody (1:10,000; ab45171, Abcam), anti-ROCK2 antibody (1:50,000; ab125025, Abcam), anti-myosin light chain kinase antibody $(1: 10,000 ;$ ab76092, Abcam), or rabbit anti-GAPDH antibody $(1: 1,000 ; \# 2118$, Cell Signaling Technology) overnight at $4{ }^{\circ} \mathrm{C}$. Protein bands were detected using an enhanced chemiluminescence reagent (ECL reagent). Band intensities were quantified using Quantity One software (Bio-Rad, USA).

\section{Statistical analysis}

The data are presented as means \pm SE. Analysis of differences between multiple groups of data were performed with one-way ANOVA followed by a post hoc Bonferroni test. For comparison between groups, a paired or unpaired Student's t-test was used. Differences were considered significant at $P<0.05$.

\section{Results}

Role of Pyk2 in the process of spontaneous colonic smooth muscle contraction

The Pyk2 protein was detected by using western blotting and the results showed that Pyk2 was expressed in colonic smooth muscle tissue (Fig. 1A). GI motility requires the complex coordination of smooth muscles and the enteric nervous system. Because the purpose of this study was to observe the relationship between Pyk2 and smooth muscle, the influence of the enteric nervous system should be excluded. As a sodium channel blocker, TTX is a drug that is commonly used to block nerve activity. The amplitudes of spontaneous contractions were obviously decreased after the administration of the 
Pyk2 inhibitor PF-431396 (Fig. 1Ba). Moreover, after pretreatment with TTX and PF-431396, the amplitudes of contractions were still decreased (Fig. 1Bb). The PF-431396-induced inhibitory effect was not affected by TTX (Fig. 1Bc). Similar results were observed when the
Rho kinase inhibitor Y-27632 was added; the contractile amplitudes were reduced, regardless of the presence of TTX or not (Figs $1 \mathrm{Ca}$ and $\mathrm{Cb}$ ). Moreover, a statistically significant difference in the relative change in amplitudes was not observed between the two conditions (Fig. 1Cc).

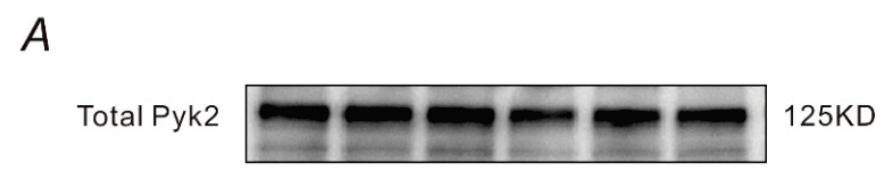

$B$

a

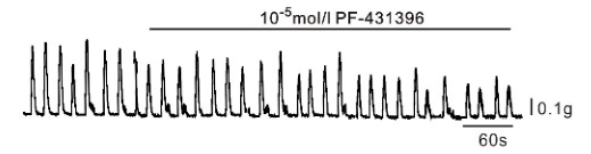

$b$
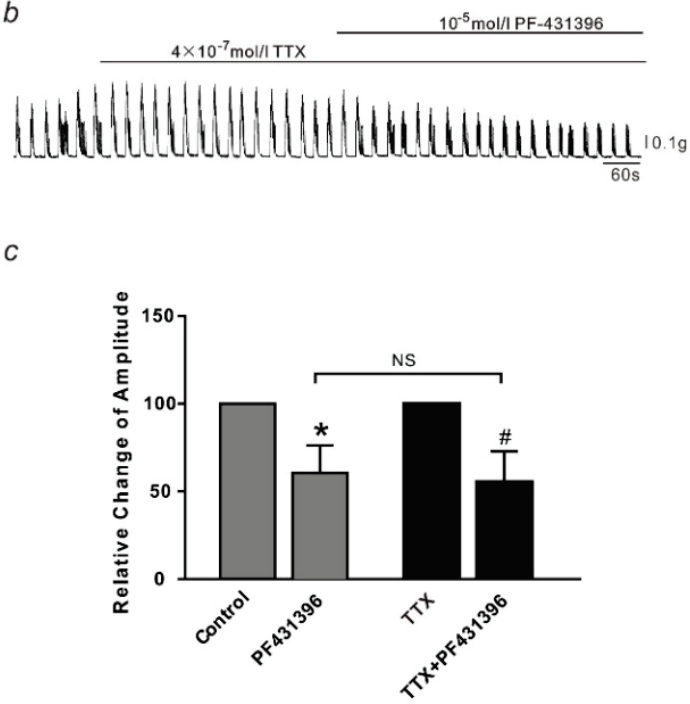

C

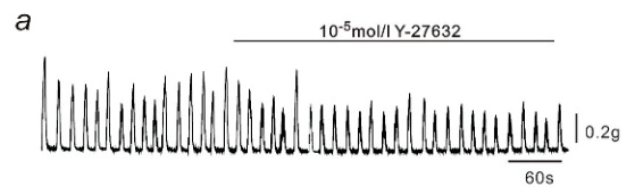

$b$

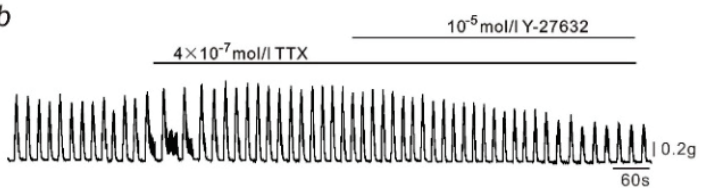

C

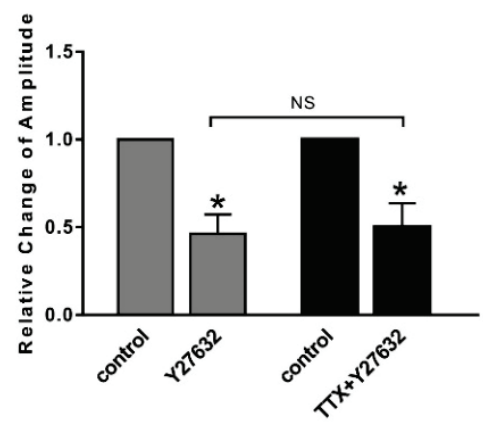

Fig. 1. Role of Pyk2 in the process of colonic smooth muscle contraction. (A) Pyk2 was expressed in the murine colonic smooth muscle tissue ( $n=6$, the $n$ values refer to the number of animals). (Ba) PF-431396 decreased the amplitude of spontaneous contractions of colonic smooth muscle strips. (Bb) PF-431396 decreased the contraction amplitude in the presence of TTX. (Bc) Summary graph showing the effect of PF-431396 (10 $\mathrm{MM}, 400 \mathrm{~s})$ on the change in the amplitude in samples treated with and without TTX. Data are presented as the means $\pm \mathrm{SE}(\mathrm{n}=6$, the $\mathrm{n}$ values refer to the number of animals; $* P<0.05$ vs. Control; $\# P<0.05$ vs. TTX; NS, not significant PF-431396 vs. TTX + PF-431396). (Ca) Y-27632 decreased the amplitude of spontaneous contractions of colonic smooth muscle strips. (Cb) Y-27632 decreased the contraction amplitude in the presence of TTX. (Cc) Summary graph showing the effect of $\mathrm{Y}-27632(10 \mu \mathrm{M}, 400 \mathrm{~s})$ on the change in the amplitude in samples treated with and without TTX. Data are presented as the means \pm $\mathrm{SE}\left(\mathrm{n}=6\right.$, the $\mathrm{n}$ values refer to the number of animals; $* P<0.05$ vs. Control; ${ }^{*} P<0.05$ vs. TTX; NS, not significant, $\mathrm{Y}-27632$ vs. TTX + $\mathrm{Y}-27632)$.

Effects of PF-431396 on the protein expression related to the contraction response in cultured CSMCs

Various kinds of interstitial cells are located in the colonic muscular layer, such as ICC and PDGFR $\alpha^{+}$ cells, which directly mediate SMCs contraction. Therefore, the cultured CSMCs were used to rule out the interactions of interstitial cells. The relative levels of intracellular proteins related to the contraction response were detected using western blotting. A significant decrease in the phosphorylation levels of Pyk2 at Tyr402 (Fig. 2A) and $\mathrm{MLC}_{20}$ phosphorylated at Ser19 were observed after cultured CSMCs were treated with PF-431396 $(10 \mu \mathrm{M})$ for $10 \mathrm{~min}$ (Fig. 2B). However, in the examination of the $\mathrm{Ca}^{2+}$-dependent pathway, the 
MLCK expression level was not affected by PF-431396 (Fig. 2C). Meanwhile, in the examination of the RhoA/ROCK pathway, PF-431396 significantly inhibited the phosphorylation of MYPT1 at Thr853 (Fig. 2D) and
ROCK2 expression (Fig. 2F), but not ROCK1 expression (Fig. 2E). Based on these findings, the role of Pyk2 was more associated with the RhoA/ROCK pathway rather than the $\mathrm{Ca}^{2+}$ dependent pathway.
A
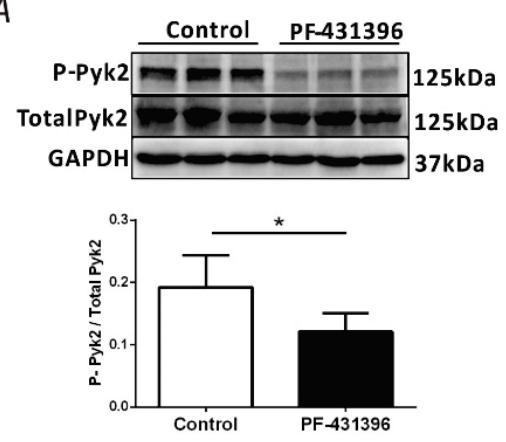

$D$
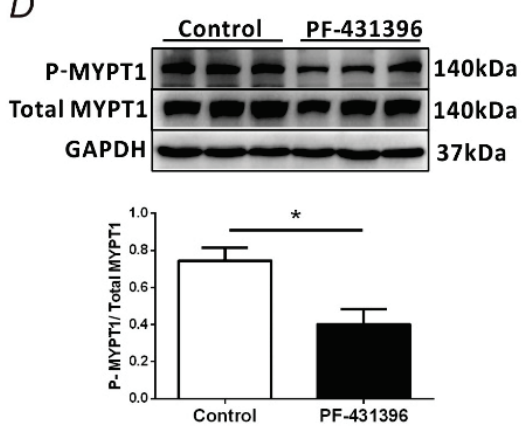

$B$
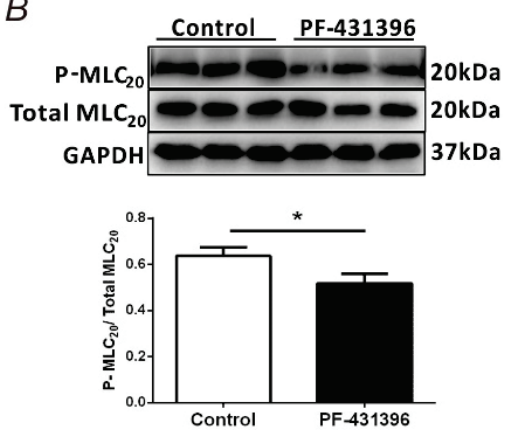

$E$
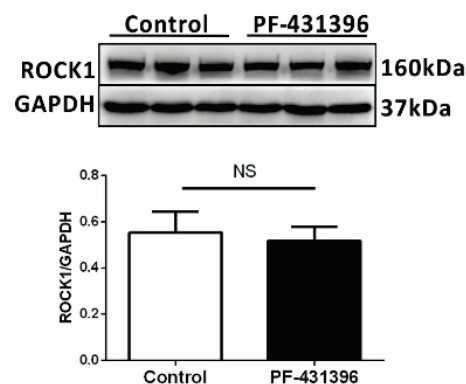

C
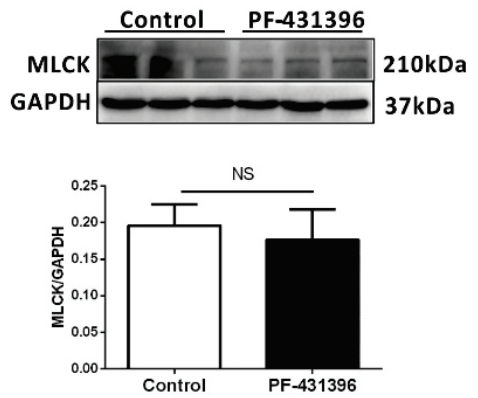

F
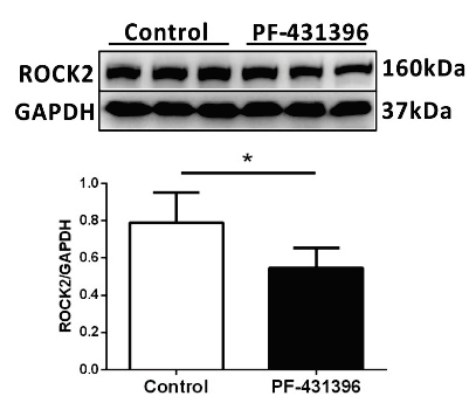

Fig. 2. Effect of PF-431396 on protein expression in cultured CSMCs. The levels of Pyk2 phosphorylated at Tyr402 (A) and MLC phosphorylated at Ser19 (B) were decreased after a 10 min incubation with PF-431396 (10 $\mu \mathrm{M})$. In the assessment of the RhoA/ROCK pathway, PF-431396 decreased the expression of the ROCK2 protein (F), but did not change ROCK1 expression (E), and decreased the level of phosphorylated MYPT1 at Thr853 (D). However, in the assessment of the Ca ${ }^{2+}$-dependent pathway, the MLCK expression level was not changed $(\mathbf{C})$. Data are presented as the means \pm SE $(n=6$, the $n$ values refer to the number of independent cultures, $* P<0.05 ; \mathrm{NS}$, not significant).

Effects of Y-27632 on contraction related protein expression in cultured CSMCs

The ROCK inhibitor Y-27632 was used to further investigate the role of Pyk2 in the RhoA/ROCK pathway. The level of Pyk2 phosphorylated at Tyr402 was weakly but not significantly increased after treatment with Y-27632 $(10 \mu \mathrm{M})$ for 20 min compared with control (Fig. 3A), whereas the level of $\mathrm{MLC}_{20}$ phosphorylated at Ser19 was decreased (Fig. 3B). Meanwhile, in the assessment of the $\mathrm{Ca}^{2+}$-dependent pathway, Y-27632 did not significantly change the MLCK expression level (Fig. 3C). However, in the examination of the RhoA/ROCK pathway, the expression levels of ROCK1, ROCK2 (Fig. 3D) and MYPT1 phosphorylated at Thr853 (Fig. 3E) were significantly decreased. Based on these findings, the activation of Pyk2 was not affected and phosphorylation of Pyk2 at Tyr402 still occurred after inhibiting the activity of ROCK.
Effects of Ach on contraction related protein expression in cultured CSMCS

Ach is the main excitatory neurotransmitter eliciting GI smooth muscle contraction. Cultured CSMCs were treated with $1 \mu \mathrm{M}$ Ach for $4 \mathrm{~min}$ to investigate whether Pyk2 and colonic smooth muscle contraction have relevant relationships in enhancing contraction responses. Ach induced significant increases in the levels of Pyk2 phosphorylated at Tyr402 (Fig. 4A) and $\mathrm{MLC}_{20}$ phosphorylated at Ser19 (Fig. 4B), as well as the levels of ROCK2 (Fig. 4C) and MYPT1 phosphorylated at Thr853 (Fig. 4D) in the RhoA/ROCK pathway; however, the ROCK1 expression level was not changed (Fig. 4C). Based on these results, the activity of Pyk2 is increased during the enhanced contractile response.

Effects of $\left[\mathrm{Ca}^{2+}\right]_{i}$ on the activation of Pyk2

An increase of $\left[\mathrm{Ca}^{2+}\right]_{\mathrm{i}}$ is the primary trigger for 

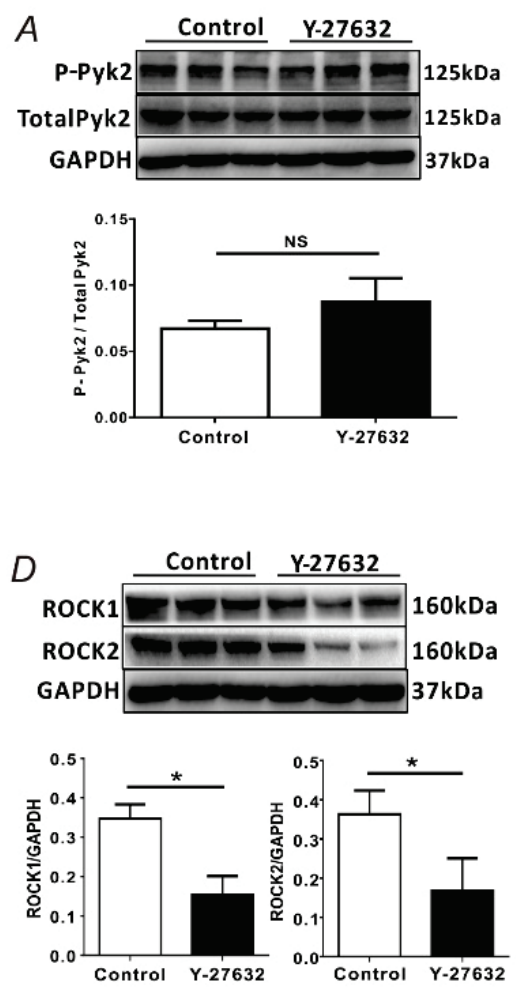
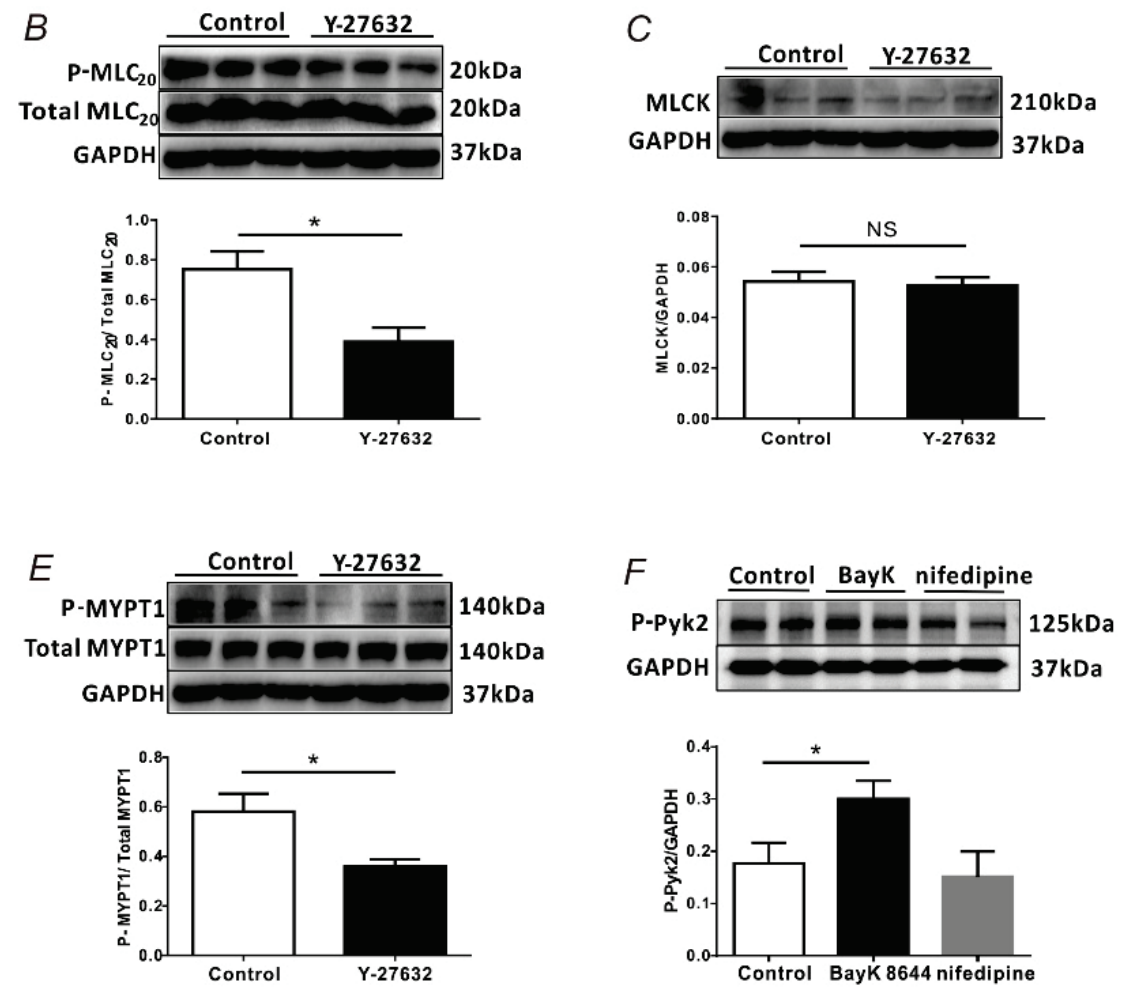
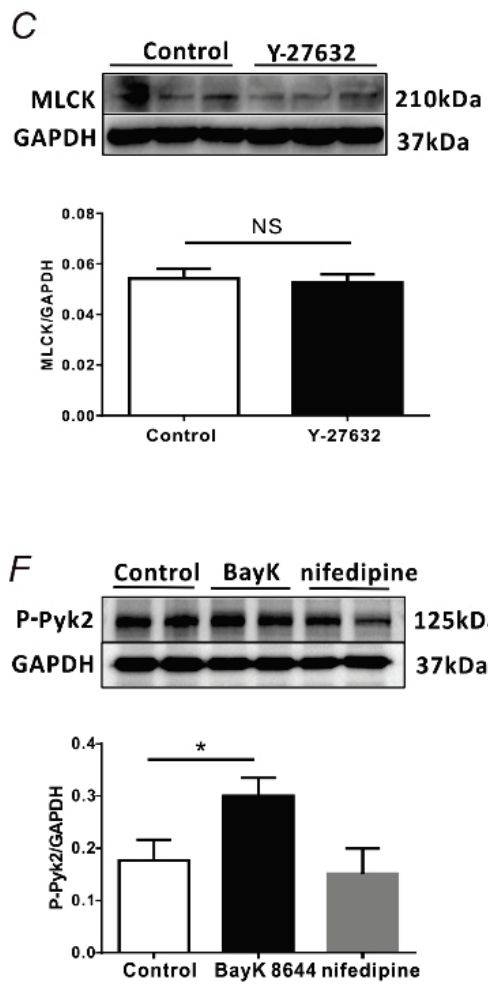

Fig. 3. Effect of Y-27632, Bay K8644 and nifedipine on protein expression in cultured CSMCs. The level of Pyk2 phosphorylated at Tyr402 (A) was increased, whereas the level of MLC phosphorylated at Ser19 (B) was decreased after a 20 min incubation with Y-27632. In the assessment of the RhoA/ROCK pathway, Y-27632 decreased the expression of the ROCK1 and ROCK2 proteins (D) and levels of phosphorylated MYPT1 at Thr853 (E). However, the MLCK expression (C) was not altered. Data are presented as the means \pm SE ( $n=6$, the $n$ values refer to the number of independent cultures, $* P<0.05$; NS, not significant). On the other hand, the level of Pyk2 phosphorylated at Tyr402 was significantly increased in CSMCs that had been treated with Bay K8644 (1 $\mu$ M) for 5 min, and decreased after treatment with nifedipine for $5 \mathrm{~min}(\mathbf{F})$. Data are presented as the means \pm SE ( $n=6$, the $n$ values refer to the number of independent cultures; $*<<0.05)$.

the contraction of GI smooth muscles. The most important channel that exerts a substantial influence on $\left[\mathrm{Ca}^{2+}\right]_{\mathrm{i}}$ is the VOCCs (Perrino 2016). CSMCs were treated with the VOCCs blocker nifedipine $(1 \mu \mathrm{M})$ or the VOCCs activator Bay K8644 $(1 \mu \mathrm{M})$ for $5 \mathrm{~min}$. Nifedipine and Bay K8644 exert different effects on the phosphorylation of Pyk2 at Tyr402. Bay K8644, but not nifedipine, significantly increased the levels of Pyk2 phosphorylated at Tyr402 in cultured CSMCs (Fig. 3F). Thus, elevated $\left[\mathrm{Ca}^{2+}\right]_{\mathrm{i}}$ triggers the phosphorylation of Pyk2 to elicit contraction responses of smooth muscle.

\section{Discussion}

In the present experiments, we have examined the relationship between Pyk2 and colonic smooth muscle motility. The main findings of this study are listed below: 1) Pyk2 was expressed in colonic smooth muscle and was activated during colonic smooth muscle contraction; and 2) during the colonic smooth muscle contraction, the activation of Pyk2 occurred prior to activation of the RhoA/ROCK signaling pathway and its involvement in the contraction response was mediated through the RhoA/ROCK pathway.

It has been reported that Pyk2 activation can accelerate the process of differentiation, proliferation and contraction of vascular smooth muscle cells (Ying et al. 2009a, Perez et al. 2011, Bhattachariya et al. 2014). VSMCs and CSMCs are both smooth muscle cells and the mechanism of contraction response in both is associated with $\mathrm{Ca}^{2+}$-dependent and $\mathrm{Ca}^{2+}$-sensitization pathways (Kuo and Ehrlich 2015); however, few studies have reported the role of Pyk2 in colonic smooth muscle contraction. It is well known that the contraction response in colonic smooth muscle in vitro is spontaneous and is associated with coordination of the enteric nervous system and smooth muscles (Murthy 2006). However, the spontaneous contraction response continues to happen even after removing the nerves and this phenomenon is associated with the smooth muscle itself, which called myogenic contraction (McCarron et al. 1997, De Godoy and Rattan 2011). In the present study, we found that 
$A$
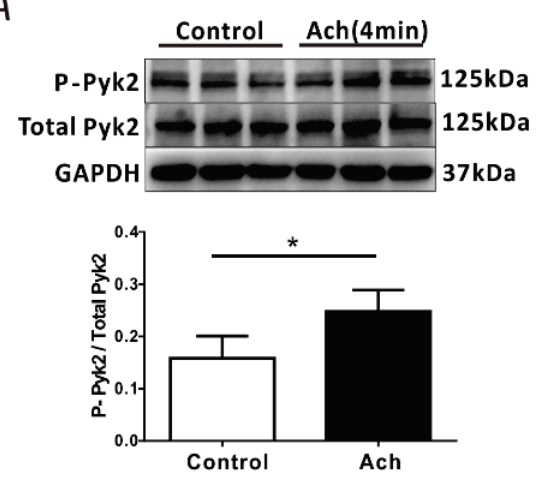

C
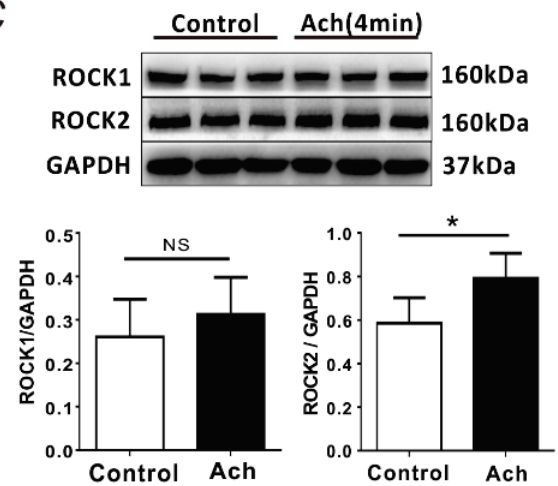
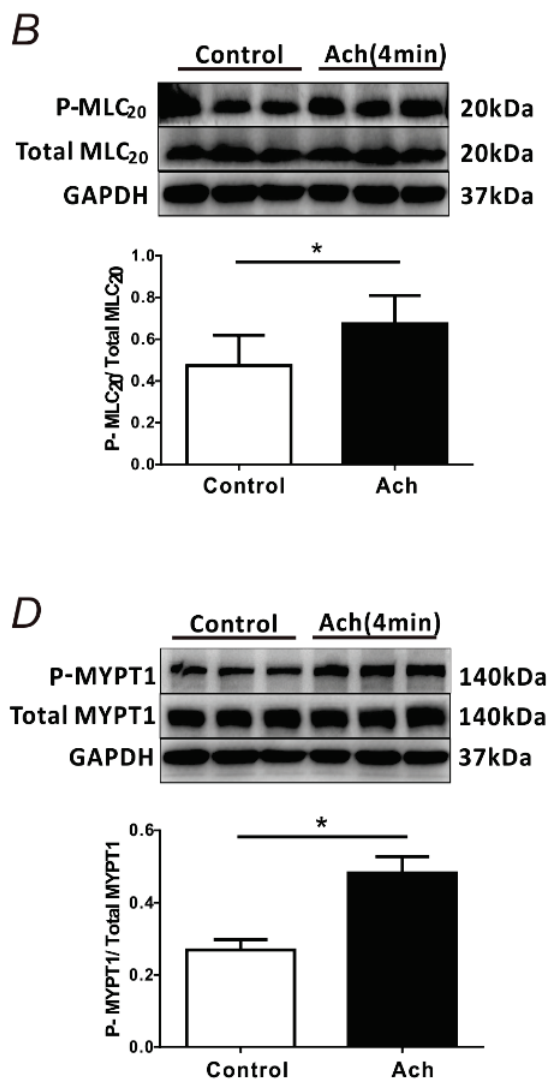

Fig. 4. Effect of Ach on protein expression in cultured CSMCs. The levels of Pyk2 phosphorylated at Tyr402 (A) and MLC phosphorylated at Ser19 (B) were increased after a 4 min incubation with Ach $(1 \mu \mathrm{M})$. In the RhoA/ROCK pathway, Ach increased the expression of the ROCK2 protein (C), did not alter ROCK1 expression (C), and increased the levels of MYPT1 phosphorylated at Thr853 (D). Data are presented as the means $\pm \mathrm{SE}$ ( $\mathrm{n}=6$, the $\mathrm{n}$ values refer to the number of independent cultures, $* P<0.05$; NS, not significant).

PF-431396 decreased the contraction response of colonic smooth muscle with or without the presence of TTX indiscriminately (Fig. 1), which showed that Pyk2 can affect the contraction response of colonic smooth muscle and this effect was not related to nerves. In addition, PF-431396 significantly decreased the expression of proteins involved in the RhoA/ROCK signaling pathway, whereas MLCK expression was not affected in the assessment of the $\mathrm{Ca}^{2+}$-dependent pathway (Fig. 2). This showed that Pyk2 is more closely associated with the $\mathrm{Ca}^{2+}$ sensitization pathway than the $\mathrm{Ca}^{2+}$ dependent pathway. ROCK has two isoforms, ROCK1 and ROCK2 (Riento and Ridley 2003), and ROCK2 participates in the contraction response of smooth muscle (Hakoshima et al. 2003, Nunes et al. 2010). We found that PF-431396 decreased ROCK2 expression whereas ROCK1 levels were not changed, and Ach increased ROCK2 expression while ROCK1 expression was not altered. However, Y-27632, a non-selective Rho kinase inhibitor, decreased the expression of both ROCK1 and ROCK2 proteins.

Next, the Rho kinase inhibitor Y-27632 was used to further investigate the relationship between the
Pyk2 and the RhoA/ROCK pathway. The results showed that Y-27632 decreased the expression of proteins related to the RhoA/ROCK signaling pathway; however, the phosphorylation level of Pyk2 at Tyr402 was not decreased (Fig. 3), which means that Pyk2 activation was not affected by Y-27632. In other words, Pyk2 may act upstream of ROCK. Because Y-27632 is a ROCK inhibitor, it only inhibits the activity of ROCK, thus leading to inhibition of the expression of downstream proteins in the RhoA/ROCK signaling pathway directly or indirectly, while the upstream proteins in the RhoA/ROCK signaling pathway may not be affected in the short term. This is also the reason why we treated CSMCs with drugs for 10 or 20 min because we wanted to observe their current protein changes. In addition, the contraction force was steadily changed at $400 \mathrm{~s}$ after using inhibitors in colonic smooth muscle strips in the experiments on isometric tension measurements. Moreover, the phosphorylation level of Pyk2 at Tyr402 was increased when treating CSMCs with Ach and Bay K8644, a VOCCs opener. These results suggest that the elevation of intracellular $\mathrm{Ca}^{2+}$ may activate Pyk2 and then 


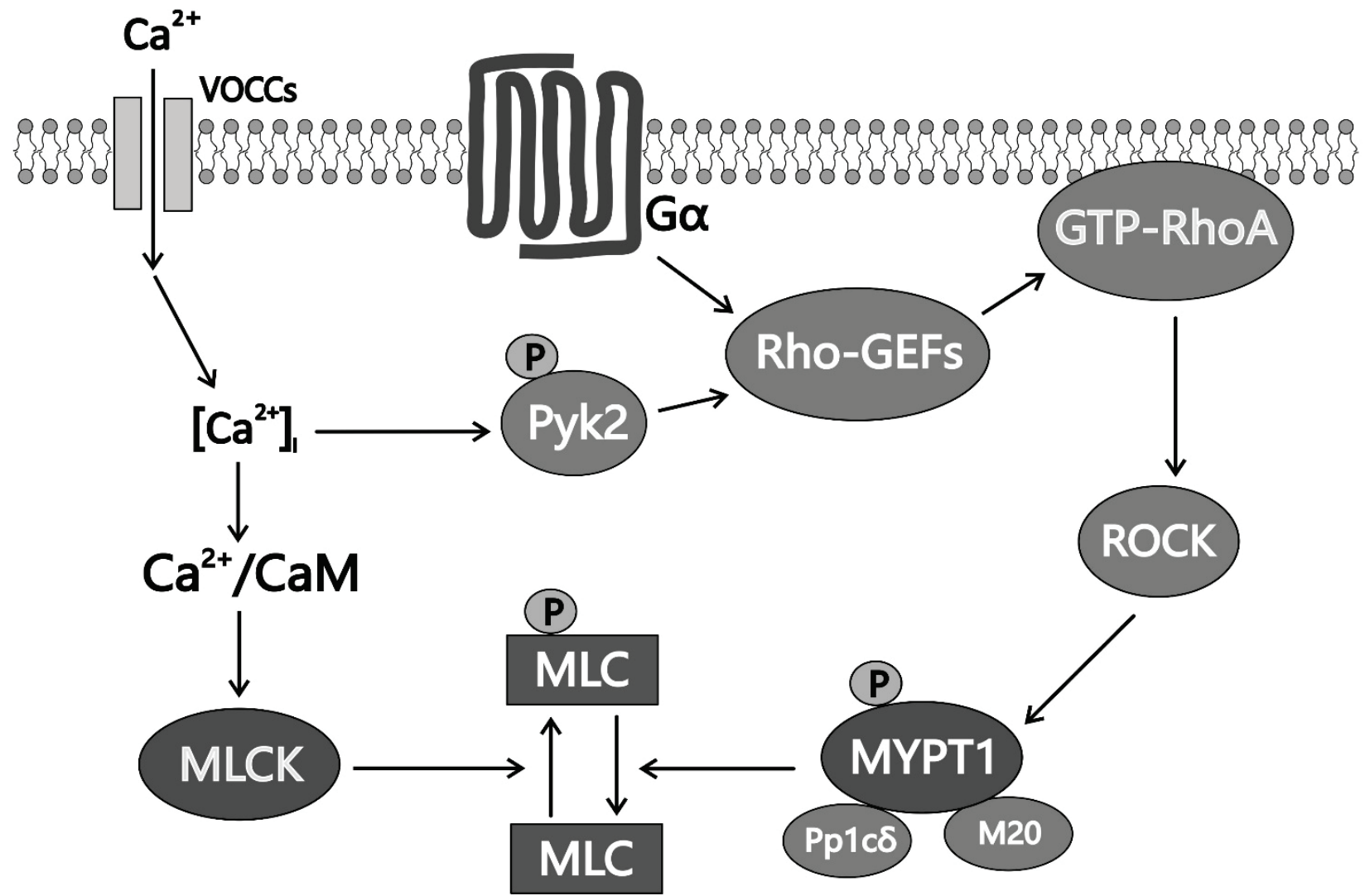

Inactive MLCP

Fig. 5. Proposed relationship between the Pyk2 and the RhoA/ROCK pathway during colon smooth muscle contraction. Depolarization of the colon smooth muscle cell plasma membrane promotes $\mathrm{Ca}^{2+}$ entry via VOCCs; then, $\mathrm{Ca}^{2+}$ binds to CaM and activates MLCK to phosphorylate MLC and facilitate cross-bridge formation, resulting in the rapid, phasic contractile response. On the other hand, $\mathrm{Ca}^{2+}$ activates Pyk2, which activates RhoA and ROCK by regulating GDP-GTP exchange factors (Rho-GEFs) and then inhibits MLCP activity by inducing MYPT1 phosphorylation. Therefore, MLC phosphorylation and force are maintained at a stable level, which is responsible for the sustained contraction.

activate the RhoA/ROCK signaling pathway and induce smooth muscle contraction.

In addition, it is well known that RhoA plays an important role in the contraction process of smooth muscle, especially in the RhoA/ROCK signaling pathway (Perrino 2016). The activity of RhoA (GTP-RhoA) is regulated by three types of interacting molecules, guanine nucleotide exchange factors (GEFs), GTPase-activating proteins (GAPs), and guanine nucleotide dissociation inhibitors (GDIs) (Bhattacharyya and Wedegaertner 2003, Rattan et al. 2003, Jin et al. 2004). In this study, although a relationship between RhoA and Pyk2 was not detected, there are many reports that have suggested that activation of Pyk2 can promote RhoA activation via RhoGEFs (Lim et al. 2008, Ying et al. 2009a), whereas ROCK is the direct downstream effector of RhoA during the contraction process (Alshboul 2015). By combining the above evidence, we identified a pathway involving Pyk2 and colonic contraction (Fig. 5). Extracellular $\mathrm{Ca}^{2+}$ entered into CSMCs through VOCCs, causing the activation of
$\mathrm{Ca}^{2+}$-dependent MLCK and increased $\mathrm{MLC}_{20}$ phosphorylation; at the same time, Pyk2, which is activated by cytosolic $\mathrm{Ca}^{2+}$, activates RhoA through RhoGEFs. GTP-RhoA activates ROCK2 to inhibit MLCP activity by phosphorylating MYPT1, which maintains the level of phosphorylated $\mathrm{MLC}_{20}$ and sustains the contraction.

In conclusion, Pyk 2 is involved in the process of colonic smooth muscle contraction via the RhoA/ROCK signaling pathway. This mechanism may be a new target for exploring the dysfunction of GI smooth muscle and may provide new insight into researching the GI motility disorders.

\section{Conflict of Interest}

There is no conflict of interest.

\section{Acknowledgements}

This work was supported by grants from the National Natural Science Foundation of China (31671192 and 31571180). 


\section{References}

ALSHBOUL O: The role of the RhoA/ROCK pathway in gender-dependent differences in gastric smooth muscle contraction. J Physiol Sci 66: 85-92, 2015.

AVRAHAM H, PARK SY, SCHINKMANN K, AVRAHAM S: RAFTK/Pyk2-mediated cellular signalling. Cell Signal 12: 123-133, 2000.

BAYER AL, HEIDKAMP MC, HOWES AL, BROWN J, BYRON KL, SAMAREL AM: Protein kinase Ce-dependent activation of proline-rich tyrosine kinase 2 in neonatal rat ventricular myocytes. $J$ Mol Cell Cardiol 35: 1121-1133, 2003.

BHATTACHARIYA A, TURCZYNSKA KM, GROSSI M, NORDSTROM I, BUCKBINDER L, ALBINSSON S, HELLSTRAND P: PYK2 selectively mediates signals for growth versus differentiation in response to stretch of spontaneously active vascular smooth muscle. Physiol Rep 2: e12080, 2014.

BHATTACHARYYA R, WEDEGAERTNER PB: Characterization of G alpha 13-dependent plasma membrane recruitment of p115RhoGEF. Biochem J 371: 709-720, 2003.

DE GODOY MA, RATTAN S: Role of rho kinase in the functional and dysfunctional tonic smooth muscles. Trends Pharmacol Sci 32: 384-393, 2011.

GROVER M, FARRUGIA M, LURKEN MS, BERNARD CE, FAUSSONE-PELLEGRINI MS, SMYRK TC, PARKMAN HP, ABELL TL, SNAPE WJ, HASLER WL, ÜNALP-ARIDA A, NGUYEN L, KOCH KL, CALLES J, LEE L, TONASCIA J, HAMILTON FA, PASRICHA PJ, NIDDK GASTROPARESIS CLINICAL RESEARCH CONSORTIUM: Cellular changes in diabetic and idiopathic gastroparesis. Gastroenterology 140: 1575-1585.e8, 2011.

HAKOSHIMA T, SHIMIZU T, MAESAKI R: Structural basis of the Rho GTPase signaling. J Biochem 134: 327-331, 2003.

HARTSHORNE DJ, ITO M, ERDÖDI F: Role of protein phosphatase type 1 in contractile functions: myosin phosphatase. J Biol Chem 279: 37211-37214, 2004.

JIN L, YING Z, WEBB RC: Activation of Rho/Rho kinase signaling pathway by reactive oxygen species in rat aorta. Am J Physiol Heart Circ Physiol 287: H1495-H1500, 2004.

KITAZAWA T, GAYLINN BD, DENNEY GH, SOMLYO AP: G-protein-mediated Ca2+ sensitization of smooth muscle contraction through myosin light chain phosphorylation. J Biol Chem 266: 1708-1715, 1991.

KUO IY, EHRLICH BE: Signaling in muscle contraction. Cold Spring Harb Perspect Biol 7: a006023, 2015.

LIM Y, LIM ST, TOMAR A, GARDELM, BERNARDTRIFILO JA, CHEN XL, URYU SA, CANETESOLER R, ZHAI J, LIN H: PyK2 and FAK connections to p190Rho guanine nucleotide exchange factor regulate RhoA activity, focal adhesion formation, and cell motility. J Cell Biol 180: 187-203, 2008.

LIPINSKI CA, LOFTUS JC: Targeting Pyk2 for therapeutic intervention. Expert Opin Ther Targets 14: 95-108, 2010.

MCCARRON JG, CRICHTON CA, LANGTON PD, MACKENZIE A, SMITH GL: Myogenic contraction by modulation of voltage-dependent calcium currents in isolated rat cerebral arteries. $J$ Physiol 498: 371-379, 1997.

MILLS RD, MITA M, NAKAGAWA J, SHOJI M, SUTHERLAND C, WALSH MP: A role for the tyrosine kinase Pyk2 in depolarization-induced contraction of vascular smooth muscle. J Biol Chem 290: 8677-8692, 2015.

MURTHY KS: Signaling for contraction and relaxation in smooth muscle of the gut. Annu Rev Physiol 68: 345-374, 2006.

NUNES KP, RIGSBY CS, WEBB RC: RhoA/Rho-kinase and vascular diseases: what is the link? Cell Mol Life Sci 67: 3823-3836, 2010.

PEREZ J, TORRES RA, ROCIC P, CISMOWSKI MJ, WEBER DS, DARLEY-USMAR VM, LUCCHESI PA: PYK2 signaling is required for PDGF-dependent vascular smooth muscle cell proliferation. Am J Physiol Cell Physiol 301: C242-C251, 2011.

PERRINO BA: Calcium sensitization mechanisms in gastrointestinal smooth muscles. J Neurogastroenterol Motil 22: 213-225, 2016.

RATTAN S, PURI RN, FAN YP: Involvement of Rho and Rho-associated kinase in sphincteric smooth muscle contraction by angiotensin II. Exp Biol Med 228: 972-981, 2003. 
RIENTO K, RIDLEY AJ: Rocks: multifunctional kinases in cell behaviour. Nat Rev Mol Cell Biol 4: 446-456, 2003.

SOMARA S, GILMONT RR, MARTENS JR, BITAR KN: Ectopic expression of caveolin-1 restores physiological contractile response of aged colonic smooth muscle. Am J Physiol Gastrointest Liver Physiol 293: G240-G249, 2007.

SOMLYO AP, SOMLYO AV: Ca2+ sensitivity of smooth muscle and nonmuscle myosin II: modulated by G proteins, kinases, and myosin phosphatase. Physiol Rev 83: 1325-1358, 2003.

SUN B, HU C, FANG H, ZHU L, GAO N, ZHU J: The effects of lactobacillus acidophilus on the intestinal smooth muscle contraction through PKC/MLCK/MLC signaling pathway in TBI mouse model. PLoS One 10: e0128214, 2015.

WANG S, CHEN C, SU K, ZHA D, LIANG W, HILLEBRANDS JL, GOOR HV, DING G: Angiotensin II induces reorganization of the actin cytoskeleton and myosin light-chain phosphorylation in podocytes through rho/ROCK-signaling pathway*. Ren Fail 38: 268-275, 2015.

WU SS, JÁCAMO RO, VONG SK, ROZENGURT E: Differential regulation of Pyk2 phosphorylation at Tyr-402 and Tyr-580 in intestinal epithelial cells: roles of calcium, Src, Rho kinase, and the cytoskeleton Cell Signal 18: 1932-1940, 2006.

YING Z, GIACHINI FR, TOSTES RC, WEBB RC: PYK2/PDZ-RhoGEF Links Ca2+ signaling to RhoA. Arterioscler Thromb Vasc Biol 29: 1657-1663, 2009a.

YING Z, GIACHINI FR, TOSTES RC, WEBB RC: Salicylates dilate blood vessels through inhibiting PYK2-mediated RhoA/Rho-kinase activation. Cardiovasc Res 83: 155-162, $2009 \mathrm{~b}$.

ZHANG S, QIU X, GU Y, WANG E: Up-regulation of proline-rich tyrosine kinase 2 in non-small cell lung cancer. Lung Cancer 62: 295-301, 2008. 